\title{
Schrittesammler kriegen keinen Diabetes
}

9 Stunden körperliche Aktivität pro Woche reduzieren das Diabetesrisiko um $60 \%$.

Fragestellung: Gibt es eine Dosis-Wirkungs-Beziehung zwischen körperlicher Aktivität und der Prävention von Typ-2-Diabetes?

Hintergrund: Wir wissen, dass körperliche Bewegung gut ist, um Diabetes vorzubeugen. Wir wissen auch, dass sie den Blutzucker senkt und dass v.a. Alltagsaktivität „gesund“ ist. Wir empfehlen sie in allen Leitlinien und unseren Patienten, $150 \mathrm{Mi}$ nuten pro Woche körperlich aktiv zu sein. Ist das alles und richtig? Wie ist der Zusammenhang zwischen körperlicher Bewegung und „Zugewinn an Gesundheit“? Welche Bedeutung hat das für die Prävention einer chronischen Erkrankung wie Typ2-Diabetes und, letztendlich, gibt es eine Dosis-Wirkungs-Beziehung, meint: Hat mehr körperliche Aktivität und Alltagsaktivität auch einen stärkeren positiven gesundheitlichen Effekt?

Originalie

Aune D, Norat T, Leitzmann M, Tonstad S, Vatten LJ. Physical activity and the risk of type 2 diabetes: a systematic review and dose-response meta-analysis. Eur J Epidemiol. 2015;30(7):529-42.
Patienten und Methoden: Die Forscher um Dagfinn Aune von der Norwegian University of Science and Technology in Trondheim in Norwegen stellten sich diese Fragen und untersuchte den Zusammenhang zwischen verschiedenen Arten körperlicher Aktivität und Typ-
2-Diabetes-Risiko. Dazu führten sie einen systematischen Review und eine Metaanalyse von publizierten Studien durch. Relevante verfügbare Studien bis März 2015 wurden in die Analyse einbezogen. Untersucht wurden in der Analyse vier Arten von Intensitäten körperlicher Aktivität: stark-, mittel- und niedrig intensive körperliche Aktivität. Zusätzlich wurde noch „Walking" als Surrogat für Alltagsaktivität gemessen.

Ergebnisse: Dabei zeigte sich, dass starke körperliche Aktivität das Diabetesrisiko um 39\% reduzierte, mittlere körperliche Aktivität um 32\% und leichte Freizeitaktivität um 26\%. 2-3 Stunden zügiges Gehen pro Woche reduzierte das Diabetesrisiko um 15\%. Die Analyse zeigte weiterhin, dass es in allen Bereichen eine inverse Assoziation von körperlicher Aktivität mit dem Diabetesrisiko gibt. Interessanterweise war das Ausmaß der Risikoreduktion, das durch Steigerung der körperlichen Aktivität erreicht werden kann, für Menschen mit zuvor niedriger Aktivität am höchsten. Dabei wurde eine Steigerung der Aktivität auf 5-7 Stunden pro Woche untersucht.

Schlussfolgerungen: Die Autoren folgern, dass jede körperliche Aktivität zur Prävention chronischer Erkrankungen, v.a. von Typ-2-Diabetes, beiträgt. Jede Aktivitätssteigerung nützt zusätzlich, wobei zu erwarten ist, dass ein kontinuierlicher Zusammenhang existiert.

\section{- Kommentar von Prof. Dr. med. Peter Schwarz}

\section{Erst ab 5000 Schritten pro Tag fängt der Nutzen richtig an}

Als hätten wir es nicht schon immer gewusst: Schrittesammler bekommen keinen Diabetes. Der vorliegende Review zeigt, dass es einen klaren Zusammenhang zwischen Diabetesprävention und gesteigerter körperlicher Aktivität gibt. Dabei ist es nicht entscheidend, Sport zu machen, sondern Freizeitaktivität und Schritte laufen sind effektiv. Das ist gut, da wir in unseren Leitlinien meist 150 Minuten Aktivität pro Woche empfehlen. Geht man nun in die Tiefe, zeigt sich, dass diese 150 Minuten (2-3 Stunden pro Woche) nur 15\% Risikoreduktion mit sich bringen. Steigert man das auf 5-7 Stunden pro Woche, sind wir im Bereich von etwa $40 \%$ Risikoreduktion.

Empfehlen wir unseren Patienten also zu wenig? Meiner Meinung nach ganz klar ja! 150 Minuten körperliche Aktivität pro Woche ist fast bedeutungslos. Andere Studien zeigen, dass ab 5.000 Schritten pro Tag ein gesundheitlicher Effekt einsetzt und alles darunter als inaktiv gilt. 150 Minuten körperliche Aktivität pro Woche entspricht etwa 3.000 Schritten am Tag und ist zu wenig. Wenn wir unsere Patienten motivieren, 10.000 Schritte täglich zu laufen (Schrittzähler und Motivations-Apps helfen, das zu erreichen) entsprächen das etwa 9 Stunden körperliche Aktivität pro
Woche und das kann, wenn man die vorliegenden Studiendaten zugrunde legt, eine bis zu 60\%ige Reduktion von Typ-2-Diabetes realisieren. Das ist toll, einfach, wirkungsvoll und machbar, wenn wir selbst davon überzeugt wären. Es liegt also an uns als Ärzten, diesen Gedanken selbst zu akzeptieren, mit gutem Beispiel im wahrsten Sinne des Wortes "voran zu gehen" und mit diesem Beispiel unsere Patienten zu motivieren, körperlich aktiv zu sein, 10.000 Schritte täglich zu laufen. Die Quintessenz dieser Studie ist - ein Schrittesammler bekommt keinen Diabetes.

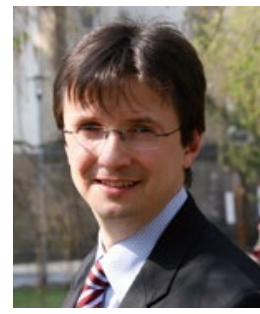

Prof. Dr. med. Peter Schwarz

Department of Medicine III

Prevention and Care of Diabetes

University of Dresden

Fetscherstr. 74, 01307 Dresden

peter.schwarz@uniklinikum-dresden.de 